\title{
The Spectrum of Epilepsies with Fixation off Sensitivity: A Case Report and Review of Literature
}

\section{Sachin Suresh-babu}

St Stephen's Hospital, New Delhi, India

Corresponding author: Dr. Sachin Suresh-babu, Senior Consultant, Neurologist, St Stephen's Hospital, New Delhi, 110054, India, Tel: 8375938480; E-mail: drsachins1@rediffmail.com

Received: Apr 12, 2017; Accepted: May 18, 2017; Published: May 22, 2017

Citation: Suresh-babu S. The Spectrum of Epilepsies with Fixation off Sensitivity: A Case Report and Review of Literature. J Neurol Neurosci 2017, 8: 3 .

\section{Abstract}

Fixation off sensistivity is a electrographic marker of occipital lobe epilepsy. The report describes a 11 year old with infrequent seizures responsive to treatment with valproate who was found to have occipital epileptiform discharges which appeared only during eye closure. The typical phenomenology of visual aura or autonomic seizures as seen in benign occipital epilepsies is not mandatory to be associated with FOS. The spectrum of various epilepsy syndrome which display this typical finding is discussed.

Keywords: Fixation off sensitivity; Occcipital lobe epilepsy

\section{Introduction}

"Fixation off sensitivity" refers to electrographic abnormalities or seizures precipitated by the suppression of visual fixation [1]. The class of epilepsies that most commonly manifests with Fixation Off Sensitivity and is almost synonymous with it is occipital lobe epilepsy. However, this electrographic finding is not specific for any of the entities within this broad and versatile category of epilepsies. There is a predilection for the younger age group [2-6]. This case report and ensuing discussion focuses on the clinical diagnosis, pathophysiology and differentials of this electro clinical syndrome.

\section{Case Report}

A 11 year girl was referred to our Neurology department for epilepsy classification and advice on management. She had a history of infrequent seizures from seven years of age, with one or two seizures in a year. She was seizure free for two years on $400 \mathrm{mg}$ of Valproate. The parents described her events as a sudden uprolling of eyes, with some extension of the neck and loss of consciousness lasting 30 seconds with frothing from the mouth. No other accompaniments like tonic posturing, myoclonic jerks, eye flickering or urinary incontinence were reported. The three episodes which they could recollect had occurred in the following circumstances: while watching television, standing in the sun for school assembly and while brushing her teeth under the light. She complained of some vague symptoms like giddiness intermittently but there were no symptoms to suggest absence or other seizure semiologies.

She was born of non-consanguineous union, full term by normal delivery in a hospital. There was no history of perinatal asphyxia or any neonatal infections. She attained normal milestones of development and was fully immunized. She was in the 5th class in her school and had an average school performance. There was no past history of any illnesses, chronic diarrhoeas, abdominal pains or failure to gain weight.

On examination, general physical, systemic and neurological examination was unremarkable. MRI brain did not reveal any focal pathologies or calcifications. During a 45 minute EEG performed with photic simulation and hyperventilation; sleep could not be obtained. The 10-20 international system with additional T1 and T2 (silverman electrodes) were used. The awake record showed $3-4 \mathrm{~Hz}, 400-500 \mu \mathrm{V}$ biphasic and at times triphasic sharp waves and spikes in the posterior head region bilaterally. In the AP longitudinal montage, phase reversals are not seen suggesting an end of chain phenomenon. The referential montages ( $C Z$ and common average reference) clearly shows the maximum negativity in bilateral posterior head region. These discharges disappeared immediately after opening of eyes thus enabling us to make a diagnosis of Fixation off Sensitivity (Figure 1). They persisted after eye opening when fixation was eliminated by use of Frenzel's glasses. Coeliac antibody panel was negative. Patient was continued on valproate and reassured regarding future course of illness. 


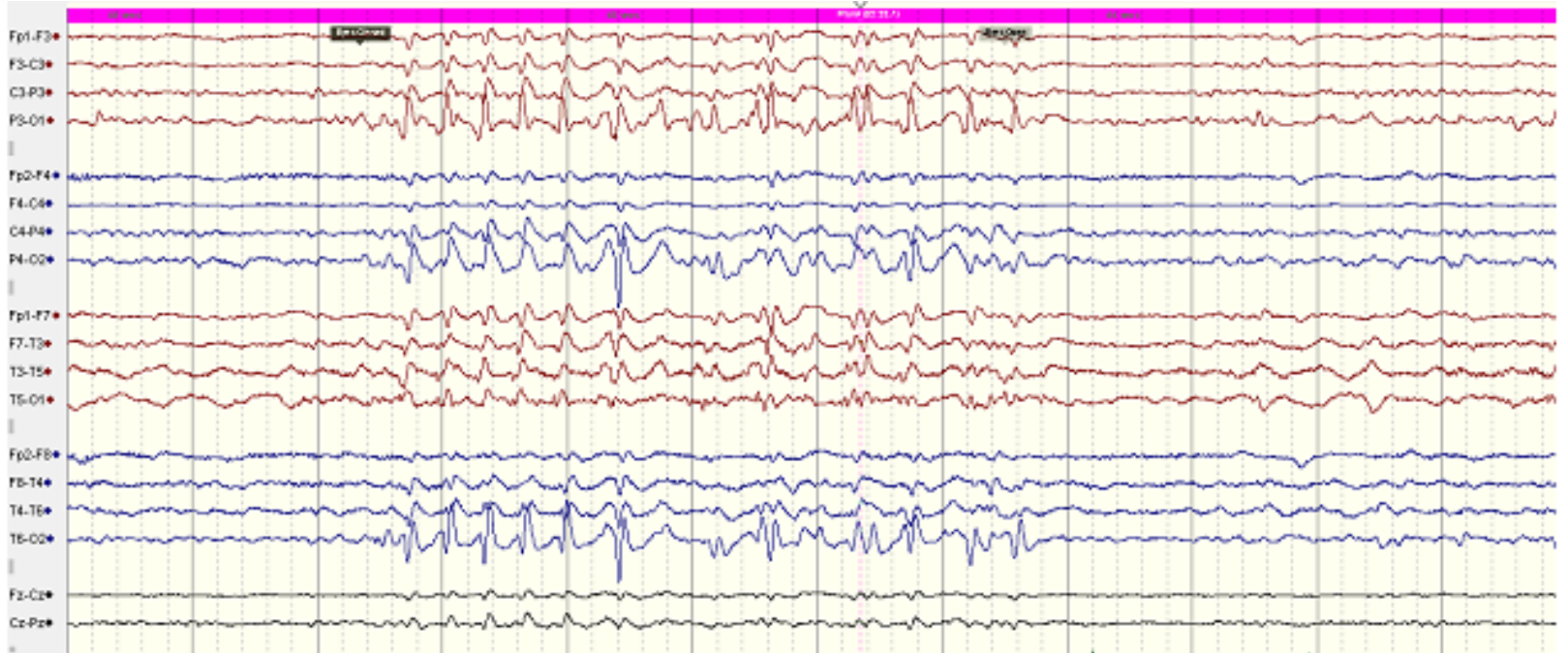

A) Bipolar AP longitudinal montage

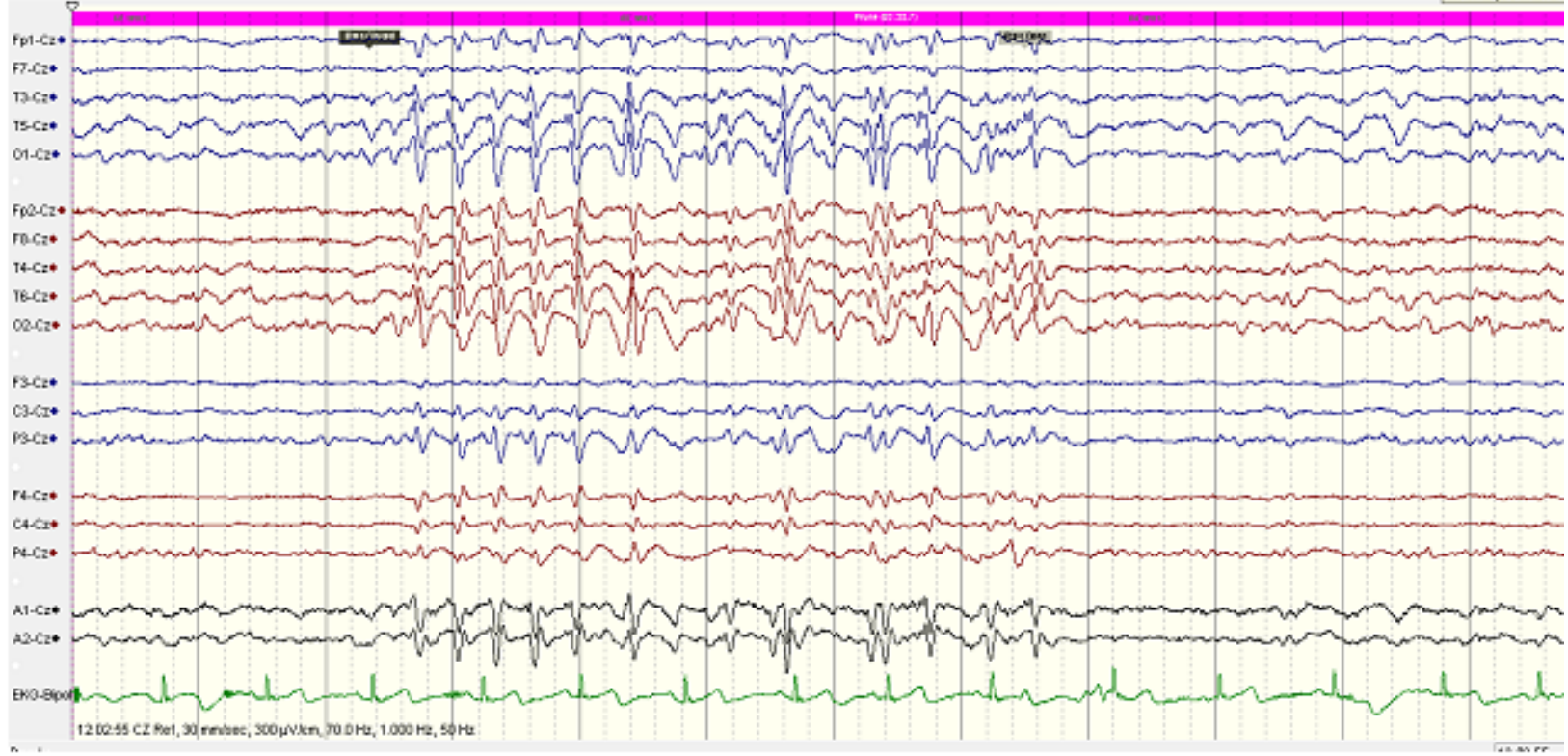

B) CZreferential montage

(12)

Figure 132 channel EEG record shows prominent 3-4 Hz, 300-400 $\mu \mathrm{V}$ mostly biphasic spike and wave discharges in the posterior head. 


\section{Discussion}

Fixation -off sensitivity is a term coined by Panayiotopoulosthat explicitly describes the phenomenon of spike waves mostly localized to the occipital or posterior head region appearing as soon as visual fixation is abolished by eye closure. Conversely opening of eyes cause this persistent train of electrical activity to completely vanish [1]. Occipital lobe epilepsies like Gestaut and Panayiotoplus syndrome, especially the former are the epilepsies most often displaying this electrographic trait. Occipital epileptogenic lesions like calcifications, dysplasias and coeliac disease are the other group of pathologies associated with FOS. In a study by Shukla et al., $72.7 \%$ of patients had symptomatic focal epilepsies which the authors attributed to the referral pattern of their high volume center [2,3]. A similar observation was made by Martinovic et al. in their analysis of 76 EEG $s$ demonstrating occipital paroxysms of which 42 showed FOS [4]. Other authors have found a lesser number of their subjects to have a demonstrable anatomical substrate on routine neuro-imaging [5].

A distinct phenotype of pure reflex epilepsy with isolated FOS has been proposed with the classical presentation occurring in females between 5-13 years manifesting with absence seizures, eyelid myoclonia, and rarely GTCS. A normal background, FOS and absence of photosensitivity are the EEG characteristics required for the diagnosis [4]. Also, various authors have opined that the spectrum of epilepsies with FOS could be more widespread than initially thought. Koutromanidis, et al. in the analysis of their videotelemetric data found both generalized and focal epilepsies contribute to the expanding repertoire of this evolving electroclinical syndrome [4]. The overwhelming contribution of the occipital lobe even in patients not fitting into the syndromic description of occipital lobe epilepsies of childhood was substantiated through the appraisal of electrical and functional imaging data by them and other investigators $[4,6,7]$.

Secondary causes were excluded by imaging and celiac work up. The typical seizure semiologies of Gastaut (visual hallucinations, andheadache) or Panayiotapoulos (Vomiting, retching, pallor and autonomic phenomena) were conspicuously absent $[1,8]$.

Jeavon's syndrome is a reflex epilepsy which can present in this age group with complex partial seizures or generalized seizures along with eye closure induced paroxysms of spikes. However, these spikes were not persistent, photosensitivity and absences with eyelid myoclonia (prototypic manifestations of Jeavon's Syndrome) were excluded after history and evaluation [9]. The confirmatory diagnosis of FOS requires demonstration of the spike wave activity after elimination of fixation by using Frenzel glasses or by recording in a dark room. Scotosensitivity, the alternate name for FOS, therefore conveys another dimension of this curious observation [1]. Photosensitivity could arguably be described as its opposite, but paradoxically, a certain degree of overlap has been observed between these two rival beauties of the electrographic world. In our patient photic stimulation with eyes open did not evoke any epileptogenic response and also did not accentuate the eye closure response. Yet another related phenomenon is the appearance of electrical discharges on fixation (inverted fixation off sensitivity) which was also excluded in the present case [10].

Our case most closely fits into the more inclusive category of disorders namely "idiopathic epilepsy with FOS" which probably represents a kind of occipital lobe epilepsy without the classical seizure semiology (either because they are not recognized by parents or they are truly absent).

The presence of FOS does not necessarily mean that the patient should have seizures. The finding although reminiscent of epileptogenic activity is not ictal in origin and its seizure predictability is borderline when compared to related phenomena like photosensitivity. In our patient, the observation was persisting even after the patient had been seizure free for four years. Hence the treatment decisions in FOS associated epilepsies, as in other benign epilepsies like BECTS should not be based on the EEG abnormalities.

\section{Conclusion}

The intent of presenting this less observed electro-clinical syndrome was to bring to attention the broader spectrum of occipital lobe epilepsies especially in the context of those that exhibit the remarkable electrical signature of FOS. The differential diagnoses have been discussed with an emphasis on the salient features of distinction which could be handy for the definitive diagnosis and classification of the syndrome.

\section{Conflicts of Interest}

None of the authors have any conflicts of interest to disclose.

\section{Acknowledgements}

We wish to acknowledge our Director of St Stephen's Hospital and the hospital management for allowing us to publish this case report.

Despite the electrical data being unequivocal, the syndromic diagnosis in the present case was challenging. Although the age group was consistent, the clinical history did not reveal the usual seizure semiologies which could have enabled us to conclude that this is "pure reflex epilepsy with isolated FOS".

\section{References}

1. Panayiotopoulos CP (1998) Fixation-off, scotosensitive, and other visual-related epilepsies. AdvNeurol 75: 139-157.

2. Kaul B, Shukla G, Goyal V, Srivastava A, Behari M (2012) Paroxysmal occipital discharges suppressed by eye opening: spectrum of clinical and imaging features at a tertiary care center in India. Neurol India 60: 461-464.

3. Koutroumanidis M, Tsatsou K, Sanders S, Michael M, Tan SV, et al. (2009) Fixation-off sensitivity in epilepsies other than the 
idiopathic epilepsies of childhood with occipital paroxysms: a 12-year clinical-video EEG study. Epileptic Disord 11: 20-36.

4. Martinovic Z (2001) Clinical correlations of electroencephalographic occipital epileptiform paroxysms in children. Seizure 10: 379-381.

5. Maher J, Ronen GM, Ogunyemi AO, Goulden KJ (1995) Occipital paroxysmal discharges suppressed by eye opening: Variability in clinical and seizure manifestations in childhood. Epilepsia 36: 52-57.

6. Fattouch J, Casciato S, Lapenta L, Morano A, Fanella M, et al. (2013) The spectrum of epileptic syndromes with fixation off sensitivity persisting in adult life. Epilepsia 7: 59-65.

7. Krakow K, Baxendale SA, Maguire EA, Krishnamoorthy ES, Lemieux $L$, et al. (2000) Fixation-off sensitivity as a model of continuous epileptiform discharges: Electroencephalographic, neuropsychological and functional MRI findings. Epilepsy Res 42 : 1-6

8. Ferrari MT, Macedo EF, Costa Neves RS, Costa LV, Tudesco IS, et al. (2013) Gastaut type idiopathic childhood occipital epilepsy. Epileptic Disord 15: 80-83.

9. Striano S, Capovilla G, Sofia V, Romeo A, Rubboli G, et al. (2009) Eyelidmyoclonia with absences (Jeavons syndrome): a welldefined idiopathic generalized epilepsy syndrome or a spectrum of photosensitive conditions? Epilepsia 5: 15-19.

10. Saadeldin IY, Matlik HN (2015) Coexistence of fixation-off sensitivity and inverted fixation-off sensitivity in a female child with Panayiotopoulos syndrome: Video-electroencephalography documentation. Epilepsy Behav Case Rep 4: 1-5. 